\title{
Anomaly constraints on deconfinement and chiral phase transition
}

\author{
Hiroyuki Shimizu and Kazuya Yonekura \\ Kavli IPMU (WPI), UTIAS, The University of Tokyo, Kashiwa, Chiba 277-8583, Japan
}

(Received 10 July 2017; published 17 May 2018)

\begin{abstract}
We study the constraints on thermal phase transitions of $\mathrm{SU}\left(N_{c}\right)$ gauge theories by using the 't Hooft anomaly involving the center symmetry and chiral symmetry. We consider two cases of massless fermions: (i) adjoint fermions and (ii) $N_{f}$ flavors of fundamental fermions with a nontrivial greatest common divisor, $\operatorname{gcd}\left(N_{c}, N_{f}\right) \neq 1$. For the first case (i), we show that the chiral symmetry restoration in terms of the standard Landau-Ginzburg effective action is impossible at a temperature lower than that of deconfinement. For the second case (ii), we introduce a modified version of the center symmetry, which we call centerflavor symmetry, and draw similar conclusions under a certain definition of confinement. Moreover, at zero temperature, our results give a partial explanation of the appearance of dual magnetic gauge groups in (supersymmetric) QCD when $\operatorname{gcd}\left(N_{c}, N_{f}\right) \neq 1$.
\end{abstract}

DOI: 10.1103/PhysRevD.97.105011

\section{INTRODUCTION AND SUMMARY}

Thermal phase transition in gauge theories is a very interesting and important subject. Theoretically, it is related to the mystery of how strong dynamics works in confinement and chiral symmetry breaking. Phenomenologically, the nature of the phase transition affects cosmological observables such as dark matter abundance. It might even provide the dark matter itself via QCD effects [1].

The standard way to study chiral symmetry restoration is as follows [2]. The quark bilinear $\Phi \sim \psi \psi$, where $\psi$ represents left-handed fermions, is believed to be the most relevant order parameter for the chiral symmetry breaking. This operator $\Phi$ is treated as the effective degrees of freedom (d.o.f.) near the critical temperature $T_{\text {chiral }}$, and the phase transition is described by a Landau-Ginzburg effective Lagrangian:

$$
\begin{aligned}
\mathcal{L}_{\text {eff }}= & \operatorname{tr}\left(\partial_{i} \Phi^{\dagger} \partial_{i} \Phi\right)+V(\Phi) \\
V(\Phi)= & c_{0} \operatorname{tr}\left(\Phi^{\dagger} \Phi\right)+c_{1}\left[\operatorname{tr}\left(\Phi^{\dagger} \Phi\right)^{2}\right]+c_{2}\left[\operatorname{tr}\left(\Phi^{\dagger} \Phi\right)\right]^{2} \\
& +c_{\text {anom }}[\operatorname{det}(\Phi)]^{t_{R}}+\cdots,
\end{aligned}
$$

where the coefficients depend on temperature $T$ and, in particular, $c_{0} \propto\left(T-T_{\text {chiral }}\right)$, and $t_{R}$ is the Dynkin index of the left-handed fermion representation. However, because of the strong coupling, it is not easy to see whether such a scenario is likely or not. It is conceivable that

Published by the American Physical Society under the terms of the Creative Commons Attribution 4.0 International license. Further distribution of this work must maintain attribution to the author(s) and the published article's title, journal citation, and DOI. Funded by SCOAP ${ }^{3}$. deconfinement happens at a lower temperature. If so, we lose intuitive reasons for treating the composite $\Phi$ as the effective elementary d.o.f. These questions may be rigorously asked in theories where center symmetry is well defined, such as adjoint QCD, QCD with imaginary baryon chemical potential [3], or QCD in the large $N_{c}$ limit.

Recently, a very remarkable paper [4] appeared which studied phase transitions in pure Yang-Mills. They used a mixed 't Hooft anomaly between the $C P$ symmetry and the 1 -form center symmetry to constrain possible phase transitions at the theta angle, $\theta=\pi$. Under reasonable assumptions about the dynamics of pure Yang-Mills, the $C P$ symmetry cannot be restored below the temperature at which the center symmetry is broken, i.e., deconfinement. See the original paper for more careful discussions.

In this paper, we point out that a similar discussion is possible for chiral symmetry. The mixed 't Hooft anomaly between chiral and center symmetry is also known [5], so we can repeat the argument of [4] when the center symmetry exists, such as $\mathrm{SU}\left(N_{c}\right)$ gauge theories with $n_{f}$ fermions in the adjoint representation. We will see that chiral symmetry restoration by (1) cannot happen below the deconfinement temperature.

When there are $N_{f}$ fermions in the fundamental representation of $\mathrm{SU}\left(N_{c}\right)$, the center symmetry no longer exists. However, we argue that there is a more subtle "symmetry" that mixes the center symmetry and flavor symmetry, by using the fact that the fermions are in the representation of $\left[\mathrm{SU}\left(N_{c}\right) \times \mathrm{SU}\left(N_{f}\right)_{V}\right] / \mathbb{Z}_{n}$ where $n:=\operatorname{gcd}\left(N_{c}, N_{f}\right)$ is the greatest common divisor. The division by $\mathbb{Z}_{n}$ leads to what we call "center-flavor symmetry". By using it, we obtain similar constraints as in the case of adjoint fermions, under a technical definition of confinement in terms of the 
quantum fluctuations of the gauge field in a confining phase. This has implications even at zero temperature. If the chiral symmetry is not broken, we need dynamical gauge fields to match the anomaly of the center-flavor symmetry. This partially explains the reason why the dual magnetic gauge groups in Seiberg's description of supersymmetric QCD appear [6,7]. In a sense, we can directly see the existence of gauge fields via the 't Hooft anomaly.

\section{SU $\left(N_{c}\right)$ WITH ADJOINT FERMIONS}

In this section, we consider $\mathrm{SU}\left(N_{c}\right)$ gauge theories with $n_{f}$ massless Weyl fermions in the adjoint representation.

\section{A. 't Hooft anomaly of chiral and center symmetry}

Here, we describe the mixed 't Hooft anomaly of chiral and center symmetry [5].

\section{Chiral symmetry}

Classically, the theory has $\mathrm{U}\left(n_{f}\right)=\left[\mathrm{U}(1)_{A} \times \mathrm{SU}\left(n_{f}\right)\right] / \mathbb{Z}_{n_{f}}$ chiral symmetry acting on the fermions. The $\mathrm{U}(1)_{A}$ is quantum mechanically broken to the anomaly-free subgroup $\mathbb{Z}_{2 N_{c} n_{f}}^{\text {axiial }}$, whose generator acts on fermions $\psi$ via

$$
\mathbb{Z}_{2 N_{c} n_{f}}^{\text {axial }}: \psi \rightarrow \exp \left(\frac{2 \pi i}{2 N_{c} n_{f}}\right) \psi
$$

Thus, the chiral symmetry of the theory is reduced to $\left[\mathrm{SU}\left(n_{f}\right) \times \mathbb{Z}_{2 N_{c} n_{f}}^{\text {axial }}\right] / \mathbb{Z}_{n_{f}}$.

The order parameter of the breaking is $\Phi_{a b}=$ $\psi_{a} \psi_{b}\left(a, b=1, \ldots, n_{f}\right)$, which behaves as

$$
\operatorname{det} \Phi_{a b}=\operatorname{const}\left(e^{i \theta}\right)^{\frac{1}{N_{c}}} .
$$

If we assume it is nonzero, there are $N_{c}$ distinct connected components in the moduli space of vacua. The generator of $\mathbb{Z}_{2 N_{c} n_{f}}^{\text {axial }}$ transformation is implemented by the theta angle rotation, $\theta \rightarrow \theta+2 \pi$, which permutes the $N_{c}$ connected components.

The continuous part $\mathrm{SU}\left(n_{f}\right)$ of the chiral symmetry is also broken by the vacuum expectation values of the matrix $\left(\Phi_{a b}\right)$, which produce Goldstone bosons at each connected component. However, the details of this breaking do not play any role in the following discussion.

\section{Center symmetry}

Because adjoint fermions transform trivially under the center $\mathbb{Z}_{N_{c}} \subset \mathrm{SU}\left(N_{c}\right)$, the theory possesses the $\mathbb{Z}_{N_{c}}$ center symmetry. The center symmetry is a typical example of 1 -form symmetry [5], which acts on line operators in the present theory.

The 1-form center symmetry can be coupled to a 2-form background $B \in H^{2}\left(X, \mathbb{Z}_{N_{c}}\right)$, where $X$ is spacetime, as follows. For a topologically nontrivial gauge bundle on a manifold $X$, we first take open covers $\left\{U_{a}\right\}_{a \in A}$ of $X$ such that the bundle is trivialized on each of $U_{a}$. They are glued by transition functions $g_{a b}$ on $U_{a} \cap U_{b}$, which we take to be $N_{c} \times N_{c}$ matrices in the fundamental representation. For $\mathrm{SU}\left(N_{c}\right)$ (as opposed to $\mathrm{SU}\left(N_{c}\right) / \mathbb{Z}_{N_{c}}$ ) bundles, they satisfy the standard consistency condition $g_{a b} g_{b c} g_{c a}=1$ if $U_{a} \cap U_{b} \cap U_{c} \neq \varnothing$. However, when we consider $\mathrm{SU}\left(N_{c}\right) / \mathbb{Z}_{N_{c}}$ bundles, we have

$$
g_{a b} g_{b c} g_{c a}=\exp \left(\frac{2 \pi i w_{a b c}^{\text {gauge }}}{N_{c}}\right), \quad w_{a b c}^{\text {gauge }} \in \mathbb{Z}_{N_{c}} .
$$

This is allowed because $\exp \left(2 \pi i w_{a b c}^{\text {gauge }} / N_{c}\right)$ is in the center $\mathbb{Z}_{N_{c}} \subset \mathrm{SU}\left(N_{c}\right)$, and hence it is trivial in $\mathrm{SU}\left(N_{c}\right) / \mathbb{Z}_{N_{c}}$. These $w_{a b c}^{\text {gauge }}$ give an element of the cohomology group:

$$
w_{2}^{\text {gauge }} \in H^{2}\left(X, \mathbb{Z}_{N_{c}}\right),
$$

which gives the obstruction to uplift the $\mathrm{SU}\left(N_{c}\right) / \mathbb{Z}_{N_{c}}$ bundle to an $\mathrm{SU}\left(N_{c}\right)$ bundle.

Including the background field, $B \in H^{2}\left(X, \mathbb{Z}_{N_{c}}\right)$ for the center 1-form symmetry corresponds to considering gauge bundles which satisfy $B=w_{2}^{\text {gauge }}$. Namely, we perform a path integral under this topological condition for the gauge field.

\section{Mixed 't Hooft anomaly}

Let us describe the mixed 't Hooft anomaly between the axial symmetry $\mathbb{Z}_{2 N_{c} n_{f}}^{\text {axial }}$ and the center symmetry [5]. Under the axial rotation (2), the standard Fujikawa's argument tells us that the path integral measure $Z(X)$ changes as

$$
Z(X) \rightarrow Z(X) \exp \left(2 \pi i \int_{X} \frac{1}{8 \pi^{2}} \operatorname{tr} F \wedge F\right),
$$

where $F$ is the gauge field strength, and the trace is in the fundamental representation.

If $B=w_{2}^{\text {gauge }}=0$, the above phase factor is trivial because the instanton number is integral. However, when we turn on the background field $B=w_{2}^{\text {gauge }} \neq 0$, we have (on a manifold like $T^{4}$ [8]) $\frac{1}{8 \pi^{2}} \int_{X} \operatorname{tr} F \wedge F=$ $-\frac{1}{2 N} \int_{X} B \wedge B \bmod 1$ and hence

$$
Z(X) \rightarrow Z(X) \exp \left(-\frac{2 \pi i}{2 N} \int_{X} B \wedge B\right) .
$$

This represents the mixed 't Hooft anomaly.

\section{Low-energy behavior}

There is an immediate consequence of the above mixed ' $t$ Hooft anomaly. It is impossible that the low-energy limit has a trivial gapped vacuum with both the chiral and center symmetries unbroken. 
By looking at the two-loop beta function, a likely scenario is as follows [9]. When $n_{f} \leq 2$, the chiral symmetry is spontaneously broken. When $n_{f}=5$, it flows to a conformal fixed point. The cases $n_{f}=3,4$ are unclear, but $n_{f}=4$ may have a conformal fixed point.

\section{B. Constraints on phase transition}

Next we use the mixed 't Hooft anomaly of the $\mathbb{Z}_{2 N_{c} n_{f}}^{\text {axial }}$ and the $\mathbb{Z}_{N_{c}}$ center symmetry to constrain the thermal phase transition. We reduce the theory along the thermal circle $S_{T}^{1}$ and obtain the effective theory on $\mathbb{R}^{3}$. The center symmetry now splits into two global symmetries. One is the 0-form center symmetry $\mathbb{Z}_{N_{c}}^{0 \text {-form }}$ acting on the Polyakov loop $L=\operatorname{tr}_{\mathbf{N}_{\mathbf{c}}} \mathrm{P} \exp \left(i \int_{S_{T}^{1}} A\right)$. The other is the 1 -form center symmetry $\mathbb{Z}_{N_{c}}^{1 \text {-form }}$ acting on spacelike Wilson loops extending along $\mathbb{R}^{3}$.

The three-dimensional effective theory still has the mixed "triangle" 't Hooft anomaly among the three symmetries $\left(\mathbb{Z}_{N_{c}}^{0 \text {-form }}\right)\left(\mathbb{Z}_{N_{c}}^{1 \text {-form }}\right)\left(\mathbb{Z}_{2 N_{c} n_{f}}^{\text {axial }}\right)$ obtained by dimensional reduction of (7). The anomaly forbids the three symmetries to be simultaneously preserved.

\section{High/low-temperature phases}

We summarize the symmetry breaking in the high/lowtemperature limit. First, we note that the fermions have an antiperiodic boundary condition along the thermal circle and have no zero modes on $\mathbb{R}^{3}$. Then, when the temperature is sufficiently high, they can be safely integrated out. The axial $\mathbb{Z}_{2 N_{c} n_{f}}^{\text {axial }}$ is unbroken.

The remaining d.o.f. consist of the $3 \mathrm{~d}$ Yang-Mills and the periodic scalars coming from the gauge field in the direction $S_{T}^{1}$. The scalars get the effective potential at the one-loop level such that the $\mathbb{Z}_{N_{c}}^{0 \text {-form }}$ is broken. The $3 \mathrm{~d}$ Yang-Mills is expected to be confined with the area law for spacelike Wilson loops. Therefore, at extremely high temperature, the $\mathbb{Z}_{N_{c}}^{0 \text {-form }}$ is broken, whereas the $\mathbb{Z}_{N_{c}}^{\text {1-form }} \times \mathbb{Z}_{2 N_{c} n_{f}}^{\text {axial }}$ is unbroken.

At very low temperature, the theory can be regarded as four dimensional. We focus on the case in which the theory is confined and the chiral symmetry is broken. Then $\mathbb{Z}_{2 N_{c} n_{f}}^{\text {axial }}$ is broken, whereas $\mathbb{Z}_{N_{c}}^{0 \text {-form }} \times \mathbb{Z}_{N_{c}}^{1 \text {-form }}$ is not.

The summary is in the following table.

\begin{tabular}{lccl}
\hline Symmetry & Low $T$ & Intermediate & High $T$ \\
\hline Center $\mathbb{Z}_{N}^{0 \text {-form }}$ & Unbroken & $?$ & Broken \\
Center $\mathbb{Z}_{N_{c}}^{1 \text {-form }}$ & Unbroken & $?$ & Unbroken \\
Axial $\mathbb{Z}_{2 N_{c} n_{f}}^{\text {axial }}$ & Broken & $?$ & Unbroken \\
\hline
\end{tabular}

\section{Inequality for $T_{\text {deconf }}$ and $T_{\text {chiral }}$}

We can define at least two critical temperatures: deconfinement temperature, $T_{\text {deconf }}$ for $\mathbb{Z}_{N_{c}}^{0 \text {-form }}$, and chiral symmetry restoration temperature, $T_{\text {chiral }}$ for $\mathbb{Z}_{2 N_{c} n_{f}}^{\text {axial }}$. We do not consider the cases with more than two critical temperatures.

Now, suppose that the chiral symmetry is restored by the Landau-Ginzburg effective action (1). Then the mixed 't Hooft anomaly implies the inequality between the two temperatures,

$$
T_{\text {deconf }} \leq T_{\text {chiral }}
$$

The reason is as follows. Suppose (8) does not hold. Then in the intermediate temperature $T_{\text {chiral }}<T<T_{\text {deconf }}$, both $\mathbb{Z}_{N_{c}}^{0 \text {-form }}$ and $\mathbb{Z}_{2 N_{c} n_{f}}^{\text {axial }}$ are unbroken. If the physics near $T_{\text {chiral }}$ is described by (1), there is no way to break the 1-form symmetry $\mathbb{Z}_{N_{c}}^{1 \text {-form }}$, and also, there is no gapless d.o.f. in $T_{\text {chiral }}<T<T_{\text {deconf }}$. This contradicts the anomaly because all of the symmetries are unbroken, and there is no d.o.f. to match the anomaly.

If (8) holds, both $\mathbb{Z}_{N_{c}}^{0 \text {-form }}$ and $\mathbb{Z}_{2 N_{c} n_{f}}^{\text {axial }}$ are broken in $T_{\text {deconf }}<T<T_{\text {chiral }}$. This is consistent with the mixed 't Hooft anomaly. However, it is a little counterintuitive to use (1) because, intuitively, the gluons and quarks are liberated in the deconfining phase $T \sim T_{\text {chiral }}>T_{\text {deconf }}$.

A lattice study [10] with $n_{f}=4$ gave a result consistent with (8). However, that result is not conclusive because the theory with $n_{f}=4$ may have a conformal fixed point $[9,11,12]$. Results in a sequence of semiclassical studies of adjoint QCD, e.g., [13-19], are consistent with our constraints.

Finally, let us mention two alternative scenarios without assuming (1). They do not require (8).

(1) There is a single first-order phase transition at $T_{c}=T_{\text {chiral }}=T_{\text {deconf }}$. When we cross the temperature $T_{c}$, the decofinement transition and the chiral symmetry restoration occur at the same time.

(2) We allow a phase with broken $\mathbb{Z}_{N_{c}}^{1 \text {-form }}$ in $T_{\text {chiral }}<T<T_{\text {deconf. Namely, we have a Higgs }}$ phase for the effective $3 \mathrm{~d}$ Yang-Mills in the intermediate temperatures as discussed in [4]. However, this scenario seems difficult in the presence of the order parameter $\Phi_{a b}$.

\section{SU $\left(\boldsymbol{N}_{\boldsymbol{c}}\right)$ WITH FUNDAMENTAL FERMIONS}

In this section, we consider $\mathrm{SU}\left(N_{c}\right)$ gauge theories with massless fermions in the fundamental and antifundamental representations $\mathbf{N}_{\mathbf{c}}+\overline{\mathbf{N}_{\mathbf{c}}}$. We assume that the flavor number $N_{f}$ and the color number $N_{c}$ have a nontrivial greatest common divisor $n:=\operatorname{gcd}\left(N_{f}, N_{c}\right) \neq 1$, which includes the case of $N_{c}=N_{f}$, such as the SU(3) QCD in the massless limit of up, down, and strange quarks.

\section{A. Center-flavor symmetry}

First, we explain a way to introduce nontrivial background fields to detect the anomaly. 
When matter fields are in the fundamental representation, it does not make mathematical sense to take transition functions as in (4) with $w_{a b c}^{\text {gauge }} \neq 0$. However, we can avoid this problem by the following trick. ${ }^{1}$ The matter fields are in the bifundamental representations $\mathbf{N}_{\mathbf{c}} \times \overline{\mathbf{N}_{\mathbf{f}}}$ of the $\mathrm{SU}\left(N_{c}\right) \times \mathrm{SU}\left(N_{f}\right)_{V}$ symmetry, where $\mathrm{SU}\left(N_{f}\right)_{V}$ is the diagonal subgroup of the $\mathrm{SU}\left(N_{f}\right)_{L} \times \mathrm{SU}\left(N_{f}\right)_{R}$ chiral symmetry. Let $n=\operatorname{gcd}\left(N_{c}, N_{f}\right)$ be the greatest common divisor of $N_{c}$ and $N_{f}$. There is a subgroup $\mathbb{Z}_{n} \subset \mathrm{SU}\left(N_{c}\right) \times$ $\mathrm{SU}\left(N_{f}\right)_{V}$ that acts trivially on the fermions. Then it is possible to consider $\left[\mathrm{SU}\left(N_{c}\right) \times \mathrm{SU}\left(N_{f}\right)_{V}\right] / \mathbb{Z}_{n}$ bundles. (See also [26] in which $\mathrm{SU}\left(N_{f}\right)_{V}$ is dynamical.)

More concretely, we consider the following gauge and flavor bundles. The flavor bundle has transition functions $h_{a b}$ satisfying

$$
h_{a b} h_{b c} h_{c a}=\exp \left(\frac{2 \pi i w_{a b c}^{\text {flavor }}}{N_{f}}\right), \quad w_{a b c}^{\text {flavor }} \in \mathbb{Z}_{N_{f}} .
$$

Then we require

$$
\frac{n}{N_{c}} w_{a b c}^{\text {gauge }}=\frac{n}{N_{f}} w_{a b c}^{\text {flavor }}:=w_{a b c} \in \mathbb{Z}_{n} .
$$

Under this condition, the fermions are put on $X$ because the total transition functions $\left(g \otimes h^{\dagger}\right)_{a b}$ satisfy $\left(g \otimes h^{\dagger}\right)_{a b} \cdot\left(g \otimes h^{\dagger}\right)_{b c} \cdot\left(g \otimes h^{\dagger}\right)_{c a}=1$. We call the "symmetry" corresponding to this background as centerflavor symmetry, although we do not give a Hilbert space interpretation.

\section{Anomaly}

We can see the existence of the anomaly of center-flavor symmetry by the following concrete setup. Compactify the spacetime to $X=S_{T}^{1} \times S_{A}^{1} \times S_{B}^{1} \times \mathbb{R}_{C}$, where $S_{T}^{1}$ will be the temporal direction (i.e., thermal circle) and $S_{A}^{1} \times S_{B}^{1} \times \mathbb{R}_{C}$ are the spatial directions. The radii of $S_{A, B}^{1}$ are taken to be much larger than that of $S_{T}^{1}$.

We introduce the flavor background along $S_{T, A, B}$ as follows $[8,27,28]$. In the direction $S_{A}^{1} \times S_{B}^{1}$, we introduce the flavor Wilson lines $\Omega_{A}$ and $\Omega_{B}$ given as

$$
\Omega_{A}=I_{N_{f} / n} \otimes \omega_{A},
$$

\footnotetext{
${ }^{1}$ The idea similar to here appeared in [20], where spinors are placed on nonspin 4-manifolds by considering $\operatorname{spin}^{c}$ structure. The gravitational background there corresponds to the flavor background here, and the $\mathrm{U}(1)$ gauge field there corresponds to the $\mathrm{SU}(N)$ gauge field here. Analogous interplay between global and gauge symmetries has also appeared in recent discussions of topological phases of matter, see, e.g., [21-23]. Formally, we are going to use the fact that the flavor symmetry acting on gauge invariant operators is $\mathrm{SU}\left(N_{f}\right)_{V} / \mathbb{Z}_{n}$, and it has the extension $1 \rightarrow \mathrm{SU}\left(N_{c}\right) \rightarrow\left[\mathrm{SU}\left(N_{c}\right) \times \mathrm{SU}\left(N_{f}\right)_{V}\right] / \mathbb{Z}_{n} \rightarrow \mathrm{SU}\left(N_{f}\right)_{V} / \mathbb{Z}_{n} \rightarrow 1$. The ideas very close to ours have appeared also in $[24,25]$.
}

$$
\Omega_{B}=I_{N_{f} / n} \otimes \omega_{B},
$$

where $I_{m}$ is the unit $m \times m$ matrix and $\omega_{A}$ and $\omega_{B}$ are $n \times n$ matrices with the commutation relation $\omega_{A} \omega_{B}=$ $e^{2 \pi i / n} \omega_{A} \omega_{B}$. Explicitly,

$$
\begin{aligned}
& \omega_{A}=\operatorname{diag}\left(1, e^{2 \pi i / n}, e^{4 \pi i / n}, \ldots, e^{2(n-1) \pi i / n}\right), \\
& \omega_{B}=\left(\delta_{i+1, j}\right)_{1 \leq i, j \leq n} .
\end{aligned}
$$

We take the flavor Wilson line in the direction $S_{T}^{1}$ to be an imaginary baryonic chemical potential $\mu_{B}[3,29]$.

$$
\Omega_{T}=e^{i \mu_{B} / N_{c}} I_{N_{f}}
$$

The flavor background is a flat connection.

For the gauge field, we have the freedom to choose their boundary conditions. Let $x_{C} \in \mathbb{R}_{C}$ be the coordinate. We impose boundary conditions at $x_{C} \rightarrow \pm \infty$ such that the gauge field approaches flat connections represented by gauge Wilson lines $W_{T}\left(x_{C}\right), W_{A}\left(x_{C}\right)$, and $W_{B}\left(x_{C}\right)$ as

$$
\begin{aligned}
& W_{A}\left(x_{C}= \pm \infty\right)=V_{A} \otimes \omega_{A} \\
& W_{B}\left(x_{C}= \pm \infty\right)=V_{B} \otimes \omega_{B} \\
& W_{T}\left(x_{C}= \pm \infty\right)=V_{ \pm \infty} \otimes I_{n},
\end{aligned}
$$

where $\omega_{A}$ and $\omega_{B}$ are the same ones as in the flavor background introduced above and $\left(V_{A}, V_{B}, V_{ \pm \infty}\right)$ are $N_{c} / n \times N_{c} / n$ unitary matrices such that $\operatorname{det} W_{A}=$ $\operatorname{det} W_{B}=\operatorname{det} W_{T}=1$, and

$$
\operatorname{det}\left(V_{ \pm \infty}\right)=\exp \left(2 \pi i m_{ \pm \infty} / n\right)
$$

for $m_{ \pm \infty} \in \mathbb{Z}_{n}$. The $V_{A}, V_{B}$, and $V_{ \pm \infty}$ commute with each other so that the gauge field is flat at infinity.

The above configurations give a nontrivial $w_{2}$ as

$$
\frac{n}{N_{f}} \int_{S_{A}^{1} \times S_{B}^{1}} w_{2}^{\text {flavor }}=\frac{n}{N_{c}} \int_{S_{A}^{1} \times S_{B}^{1}} w_{2}^{\text {gauge }}=1 \quad \bmod n .
$$

Due to this $w_{2}$, there are fractional instantons in $X$ with instanton charge $\left(m_{+\infty}-m_{-\infty}\right) / n \bmod 1$ [30] (see also [31]). We remark that explicit instanton solutions are not at all necessary for our discussion, and only the topological data are important. Then, the Atiyah-Patodi-Singer (APS) index theorem states (for generic $V_{A}, V_{B}, V_{ \pm \infty}, \mu_{B}$ ) that there are fermion zero modes such that under the $\mathbb{Z}_{2 N_{f}}^{\text {axial }}$ axial rotation

$$
\mathbb{Z}_{2 N_{f}}^{\text {axial }}: \psi \rightarrow e^{\frac{2 \pi i}{2 N_{f}}} \psi
$$

the path integral measure $Z(X)$ gets a phase factor: 


$$
Z(X) \rightarrow Z(X) \cdot \exp \left(2 \pi i\left(m_{+\infty}-m_{-\infty}\right) / n\right) .
$$

This is the key anomaly for our purposes.

\section{B. Constraints by anomaly}

We would like to discuss some consequences of the anomaly (19).

\section{Thermal phase transition}

Because of the anomaly (19), there are constraints on phase transitions. First, let us discuss the case of a specific value of $\mu_{B}$. The fundamental fermions are coupled to the total Wilson line $W_{T} \otimes \Omega_{T}^{\dagger}$. Now, the effect of center symmetry action $W_{T} \rightarrow e^{-2 \pi i / N_{c}} W_{T}$ can be compensated by the shift $\mu_{B} \rightarrow \mu_{B}-2 \pi$. By combining parity in the $S_{T}$ direction, $\mu_{B} \rightarrow-\mu_{B}$, we find that there is a $\mathbb{Z}_{2}$ symmetry if $\mu_{B}=\pi[3,29]$. This $\mathbb{Z}_{2}$ acts on the Polyakov loop, $L=$ $\operatorname{tr}_{\mathbf{N}_{\mathrm{c}}} W_{T}$ as $L \rightarrow\left(e^{-2 \pi i / N_{c}} L\right)^{*}$, where $e^{-2 \pi i / N_{c}}$ comes from the center symmetry action and the complex conjugate comes from the parity flip on $S_{T}^{1}$. Thus, this is a symmetry whose order parameter is the Polyakov loop, and as we discuss below, it is broken at high temperature and unbroken at low temperature. Therefore, this $\mathbb{Z}_{2}$ can be used for a rigorous definition of deconfinement/confinement phases at $\mu_{B}=\pi$, just as the $\mathbb{Z}_{N_{c}}^{0 \text {-form }}$ symmetry of the adjoint fermion theory.

At high temperature, the $\mathbb{Z}_{2}$ is spontaneously broken just by standard perturbative computation at finite temperature, and the minima of the effective potential are at $W_{T}=I_{N_{c}}$ and $e^{2 \pi i / N_{c}} I_{N_{c}}$, which are related by $\mathbb{Z}_{2}$. Now let us take the boundary conditions as $m_{-\infty}=0$ and $m_{+\infty}=1$. These two values are in the two vacua related by the spontaneously broken $\mathbb{Z}_{2}$. Then $m_{-\infty}=0$ and $m_{+\infty}=1$ indicate that the gauge configuration approaches these two vacua at $x_{C} \rightarrow \pm \infty$. The domain wall interpolating the two vacua is the fractional instanton.

At very low temperature, we can see that $\mathbb{Z}_{2}$ is unbroken as follows. If it were broken, then by changing the value of $\mu_{B}$ from $\pi-\epsilon$ to $\pi+\epsilon$ for an infinitesimal $\epsilon$, there would be a phase transition from one phase to another which are related by $\mathbb{Z}_{2}$. However, the $\mu_{B}$ is coupled to the baryon number and all of the particles having nonzero baryon numbers are heavy. Therefore, the change of $\mu_{B}$ from $\pi-\epsilon$ to $\pi+\epsilon$ cannot change the dynamics of low-energy physics (i.e., the effective theory of pions) if the temperature is significantly lower than the lowest baryon mass, and hence, there are no phase transitions associated with the assumed spontaneously broken $\mathbb{Z}_{2}$ symmetry. Therefore, the $\mathbb{Z}_{2}$ should not be broken. This consideration is in agreement with the numerical results in [29] (see Fig. 3 of that paper).

The fact that $\mathbb{Z}_{2}$ is unbroken at low temperatures means that $W_{T}$ is well-fluctuating and the vacuum state has an overlap with any value of $m_{ \pm \infty}$. So the boundary conditions $m_{-\infty}=0$ and $m_{+\infty}=1$ become irrelevant at low temperature.

Therefore, we have the following situation;

\begin{tabular}{lccc}
\hline & $\mathbb{Z}_{2 N_{f}}^{\text {axial }}$ & $\mathbb{Z}_{2}$ & Source of anomaly \\
\hline Low $T$ & Broken & Unbroken & $\mathbb{Z}_{2 N_{f}}^{\text {axial breaking }}$ \\
Intermediate & $?$ & $?$ & $?$ \\
High $T$ & Unbroken & Broken & Fractional instanton \\
\hline
\end{tabular}

Comparison with the case of adjoint fermions is done as $\mathbb{Z}_{2 N_{c} n_{f}}^{\text {axial }} \leftrightarrow \mathbb{Z}_{2 N_{f}}^{\text {axial }}, \mathbb{Z}_{N_{c}}^{0 \text {-form }} \leftrightarrow \mathbb{Z}_{2}$, and $\mathbb{Z}_{N_{c}}^{\text {1-form }}$ correspond to the center-flavor background $\Omega_{A, B}$ described above. The anomaly (19) excludes the possibility that the phase transition is simply described by (1) below the deconfinement temperature at which the $\mathbb{Z}_{2}$ symmetry is broken.

Next, we give a speculative discussion about the case of $0 \leq \mu_{B}<\pi$. Even though there is no $\mathbb{Z}_{2}$ symmetry, we may still define confinement in the following technical sense. Our spacetime is Euclidean, so we can regard $\mathbb{R}_{C}$ as a time direction and find a ground state $|\Omega\rangle$ on $S_{T}^{1} \times S_{A}^{1} \times S_{B}^{1}$. (This idea is well-known in the case of 2d CFT) The boundary conditions $m_{ \pm \infty}$ also define physical states $\left|m_{ \pm \infty}\right\rangle .^{2} \mathrm{We}$ define confinement as the statement that $\langle m=1 \mid \Omega\rangle \neq 0$ as well as $\langle m=0 \mid \Omega\rangle \neq 0$. The $|m=0\rangle$ is expected to always have overlap with $|\Omega\rangle$ for $\left|\mu_{B}\right| \leq \pi$. In the presence of $\mathbb{Z}_{2}$, we have $\langle m=1 \mid \Omega\rangle=\langle m=0 \mid \Omega\rangle$ if $\mathbb{Z}_{2}$ is unbroken in $|\Omega\rangle$. So this condition $\langle m=1 \mid \Omega\rangle \neq 0$ is a generalization of the above case of $\mu_{B}=\pi$ to any value of $\mu_{B}$. Deconfinement means that $\langle m \mid \Omega\rangle=0$ for $m \neq 0$. This criterion of (de) confinement might be supported by an analytical picture of confinement [30]. Intuitively, confinement means (see, e.g., [8]) that the gauge field and, in particular, $W_{T}$ is quantum mechanically well fluctuating. Then $|\Omega\rangle$ is a superposition of states with all possible values of $W_{T}$. Because $m$ is related to the values of $W_{T}$ [see (17)], we expect $\langle m \mid \Omega\rangle \neq 0$ for all $m$ in a confining phase. On the other hand, it is localized near $W_{T}=I_{N_{c}}$ in a deconfining phase. We leave it as a future work to study more details of this criterion.

If the theory confines in the above technical sense, we do not have a domain wall interpolating $x_{C}=+\infty$ and $x_{C}=-\infty$. Thus, the anomaly cannot be matched by a domain wall (i.e., fractional instanton), and hence, the chiral symmetry must be broken. Then the anomaly constraint (19) works in the same way as that in the case of $\mu_{B}=\pi$. Again, (1) is impossible below the deconfinement temperature.

Let us make another remark about the effects of $\mu_{B}$. Because the baryon charge of quarks is $1 / N_{c}$, the $\mu_{B}$ is coupled to quarks via $\mu_{B} / N_{c}$. Therefore, in large $N_{c}$ counting, the effect of $\mu_{B} / N_{c}$ is a subleading effect. The

\footnotetext{
${ }^{2}$ For fermions, we need to impose APS boundary conditions for the APS index theorem to work. They have natural Hilbert space interpretations [32].
} 
inequality $T_{\text {deconf }} \leq T_{\text {chiral }}$ is indeed satisfied [33] in a holographic QCD model [34]. For recent numerical studies at $N_{c}=3$, see, e.g., [35,36].

\section{Dual magnetic gauge group}

The anomaly (19) has implications at zero temperature. Let us consider supersymmetric QCD. One of the most remarkable phenomena is the appearance of a dual magnetic gauge group in Seiberg's description of those theories $[6,7]$. The 't Hooft anomaly of the usual chiral symmetries was important for those results.

We would like to add one more evidence that may give some new insight. Suppose that the chiral symmetry is unbroken. Now, if the theory contains only scalars and fermions in low energy after confinement, it cannot match the anomaly (19). The key fact here is that the anomaly exists even when the flavor background is flat and that the flat background itself does not produce any fermion zero modes. Therefore, the anomaly (19) detects the existence of dual magnetic gauge fields.

Now let us apply anomaly matching of (19) to supersymmetric QCD.

(i) $N_{f} \leq N_{c}$ : the chiral symmetry is broken. ${ }^{3}$

(ii) $N_{f}=N_{c}+1$ : the chiral symmetry is unbroken, and the theory is confined. In this case, we have $\operatorname{gcd}\left(N_{c}, N_{c}+1\right)=1$, so the anomaly (19) vanishes. Thus, a dual magnetic gauge group need not appear.

(iii) $N_{c}+2 \leq N_{f}<3 N_{c}$ : the chiral symmetry is unbroken, and a dual magnetic gauge group appears $\mathrm{SU}\left(N_{f}-N_{c}\right)$. This satisfies the anomaly

\footnotetext{
${ }^{3}$ Baryonic branch for $N_{f}=N_{c}$ is killed by at least one of the boundary conditions or by $\mu_{B}$.
}

matching of (19) because $\operatorname{gcd}\left(N_{c}, N_{f}\right)=$
$\operatorname{gcd}\left(N_{f}-N_{c}, N_{f}\right) .{ }^{4}$

Our anomaly argument does not rely on supersymmetry at all, so it can also constrain a magnetic gauge group in nonsupersymmetric QCD. It would be interesting to apply these constraints to ideas such as hidden local symmetry [37] (see also [38]).

\section{ACKNOWLEDGMENTS}

The authors would like to thank Y. Kikukawa, T. Misumi, K. Mukaida, S. Shirai, S. Sugimoto, and T. T. Yanagida for helpful discussions. The work of K. Y. is supported in part by the WPI Research Center Initiative (MEXT, Japan) and is also supported by JSPS KAKENHI Grant-in-Aid (17K14265). The work of H. S. is partially supported by the Programs for Leading Graduate Schools, MEXT, Japan, via the Leading Graduate Course for Frontiers of Mathematical Sciences and Physics, and also supported by JSPS Research Fellowship for Young Scientists.

Note added.-Recently, we became aware of that two closely related papers [39,40] appeared. In [39], the authors obtained the inequality (8) for adjoint QCD by using the same anomaly as ours. In [40], the authors introduced the new order parameter for QCD by using the mixing of the center and flavor symmetry.

\footnotetext{
${ }^{4}$ Here, we need to assume that the quantities $m_{ \pm \infty} \in \mathbb{Z}_{n}$ specifying the boundary conditions are also matched under the Seiberg duality. However, the precise forms of $\left(V_{A}, V_{B}, V_{ \pm \infty}\right)$ appearing in the boundary conditions need not be matched under the duality. Only the topological data $m_{ \pm \infty}$ matters.
}

[1] E. Witten, Phys. Rev. D 30, 272 (1984).

[2] R. D. Pisarski and F. Wilczek, Phys. Rev. D 29, 338 (1984).

[3] A. Roberge and N. Weiss, Nucl. Phys. B275, 734 (1986).

[4] D. Gaiotto, A. Kapustin, Z. Komargodski, and N. Seiberg, J. High Energy Phys. 05 (2017) 091.

[5] D. Gaiotto, A. Kapustin, N. Seiberg, and B. Willett, J. High Energy Phys. 02 (2015) 172.

[6] N. Seiberg, Nucl. Phys. B435, 129 (1995).

[7] K. A. Intriligator and N. Seiberg, Nucl. Phys. B, Proc. Suppl. 45, 1 (1996).

[8] E. Witten, Adv. Theor. Math. Phys. 5, 841 (2001).

[9] M. Shifman, Mod. Phys. Lett. A 28, 1350179 (2013).

[10] F. Karsch and M. Lutgemeier, Nucl. Phys. B550, 449 (1999).

[11] L. Del Debbio, Proc. Sci., Lattice2010 (2014) 004.
[12] T. DeGrand, Y. Shamir, and B. Svetitsky, Phys. Rev. D 88, 054505 (2013).

[13] P. Kovtun, M. Unsal, and L. G. Yaffe, J. High Energy Phys. 06 (2007) 019.

[14] M. Unsal, Phys. Rev. Lett. 100, 032005 (2008).

[15] M. Unsal, Phys. Rev. D 80, 065001 (2009).

[16] E. Poppitz and M. Unsal, J. High Energy Phys. 09 (2009) 050.

[17] M. M. Anber, E. Poppitz, and M. Unsal, J. High Energy Phys. 04 (2012) 040.

[18] T. Misumi and T. Kanazawa, J. High Energy Phys. 06 (2014) 181.

[19] M. M. Anber and E. Poppitz, J. High Energy Phys. 10 (2015) 051.

[20] E. Witten, Math. Res. Lett. 1, 769 (1994). 
[21] N. Seiberg and E. Witten, Prog. Theor. Exp. Phys. 2016, 12C101 (2016).

[22] Y. Tachikawa and K. Yonekura, Prog. Theor. Exp. Phys. (2016) 093B07.

[23] E. Witten, Phys. Rev. B 94, 195150 (2016).

[24] F. Benini, P.-S. Hsin, and N. Seiberg, J. High Energy Phys. 04 (2017) 135.

[25] Z. Komargodski, A. Sharon, R. Thorngren, and X. Zhou, arXiv: 1705.04786.

[26] Y. Tanizaki and Y. Kikuchi, J. High Energy Phys. 06 (2017) 102.

[27] G. 't Hooft, Nucl. Phys. B153, 141 (1979).

[28] E. Witten, Nucl. Phys. B202, 253 (1982).

[29] P. de Forcrand and O. Philipsen, Nucl. Phys. B642, 290 (2002).

[30] M. Yamazaki and K. Yonekura, J. High Energy Phys. 07 (2017) 088.
[31] K. Ohmori, H. Shimizu, Y. Tachikawa, and K. Yonekura, J. High Energy Phys. 07 (2015) 014.

[32] K. Yonekura, J. High Energy Phys. 09 (2016) 022.

[33] O. Aharony, J. Sonnenschein, and S. Yankielowicz, Ann. Phys. (Amsterdam) 322, 1420 (2007).

[34] T. Sakai and S. Sugimoto, Prog. Theor. Phys. 113, 843 (2005).

[35] C. Bonati, M. D'Elia, M. Mariti, M. Mesiti, F. Negro, and F. Sanfilippo, Phys. Rev. D 93, 074504 (2016).

[36] O. Philipsen and C. Pinke, Phys. Rev. D 93, 114507 (2016).

[37] M. Bando, T. Kugo, S. Uehara, K. Yamawaki, and T. Yanagida, Phys. Rev. Lett. 54, 1215 (1985).

[38] Z. Komargodski, J. High Energy Phys. 02 (2011) 019.

[39] Z. Komargodski, T. Sulejmanpasic, and M. Unsal, Phys. Rev. B 97, 054418 (2018).

[40] A. Cherman, S. Sen, M. Unsal, M. L. Wagman, and L. G. Yaffe, Phys. Rev. Lett. 119, 222001 (2017). 